\title{
The Imaging Experiment and Its Analyses for Underwater Imaging
}

\section{Jiang Xingfang ${ }^{1,2, a^{*}}$, Ren Weidong ${ }^{1, b}$, Sun Chenyang ${ }^{1, c}$}

1) School of Mathematics and Physics, Changzhou University, Changzhou213164, China,

2) State Key Laboratory of Satellite Ocean Environment Dynamics, Second Institute of Oceanography, Hangzhou 310012, China

axfjiang@cczu.edu.cn, bwdren@cczu.edu.cn, ccysun@cczu.edu.cn

*Corresponding author

Keywords: Underwater Imaging; fitting curve; Exponential function; polynomial function

\begin{abstract}
For finding the changing law that the interaction between the photons and the water, the experiment has been finish and the result has been analyzed. Two half circles of them one is black and another is white are in different depth in water and the images are took. The results are shown that the object is just right distinguished when the depth of object in water is two times its diameter. The fitting curves of the brightness and the contrast with exponential function is tolerated. However, the fitting curve of the product of the brightness and the contrast with exponential function is not tolerated. The fitting curve is good with polynomial function.
\end{abstract}

\section{Introduction}

The oceanic resource and its button resource are developing with the land resource has been developed highly. At the same time the observation research for the ocean such as the global carbon circulation, global climate change has been put forwarded ${ }^{[1]}$. The underwater detection technique is the third hot points with space technology and oceanic technology ${ }^{[2]}$. The airborne ocean laser detection system consists of the laser, the optical device, a receiver processor, and the power supply device. The High instantaneous power pulse is from the laser with blue-green. The laser would be to the target through the atmosphere and the water transmission medium. Part of them is reflected and is received by the receiver. The underwater detection technique is used to the searching for assistance, the underwater scout, and the object identification ${ }^{[3-4]}$. The reference 5 has introduced for analyses the law of underwater imaging based on angular distribution estimation method of multiply scattered photons. This topic is based on an experiment and the experiment is with two half circle. The one of the half circle is black and another is white. The experimental object is a circle that concluded two half circle. The experimental object is into water in different depth and the images are taken.

\section{Theory}

The interaction between the photons and water. The intensity of the incident light is $I_{0}$, the depth of the medium is $d$, as shown in Figure1. It took the infinitesimal length $r \sim r+\mathrm{d} r$, and the corresponding intensity is $I \sim I+\mathrm{d} I$. Here is $\mathrm{d} r>0$ and $\mathrm{d} I>0$. Then is $(I+\mathrm{d} I)-I=-\alpha I \mathrm{~d} r, \int_{I_{9}}^{I} \frac{\mathrm{d} I}{I}=\int_{0}^{r}-\alpha \mathrm{d} r$. The integral result is $I=I_{0} \mathrm{e}^{-\alpha r}$. The transmission intensity of the light is as exponential function.

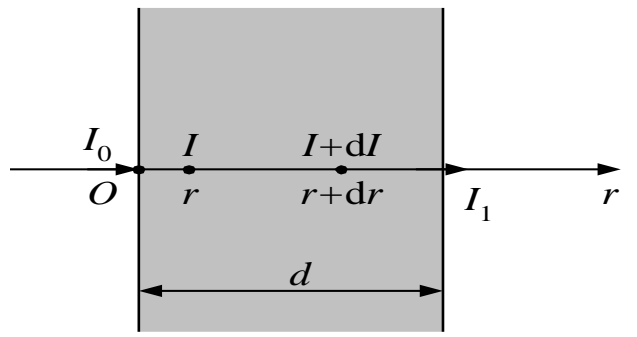

Figure 1. The transmission intensity through the medium 
The image parameters. The brightness values of each pixel with software of Matlab could be read. The programs for calculation of the brightness, contrast, and the product of the brightness and the contrast could be finished with Matlab. For an image the brightness values of each pixel $i=1,2,3, \cdots, n$ are $I_{1}, I_{2}, I_{3}, \cdots, I_{n}$ and expresses of the brightness, contrast, and the product of the brightness and the contrast as

$$
\text { bright }=\frac{\sum_{i=1}^{n} I_{i}}{n}, \text { contrast }=\sqrt{\frac{\sum_{i=1}^{n}\left(I_{i}-\text { bright }\right)^{2}}{n}}, \mathrm{~b} * \mathrm{c}=\text { bright } * \text { contrast }=\frac{\sum_{i=1}^{n} I_{i}}{n} \sqrt{\frac{\sum_{i=1}^{n}\left(I_{i}-\text { bright }\right)^{2}}{n}}
$$

The fitting curve of exponential function

For an array that is $\left(x_{1}, y_{1}\right),\left(x_{2}, y_{2}\right), \ldots,\left(x_{2}, y_{2}\right)$. The fitted curve of exponent function is as

$$
y=A \mathrm{e}^{B x}
$$

It took the logarithm $\ln y=B x+\ln A$, Note $z=\ln y, C=\ln A$, and $z=B x+C$. The differences between the experimental point and the fitted curve of exponent function are $\delta_{1}=z_{1}-\left(B x_{1}+C\right), \delta_{2}=z_{2}-\left(B x_{2}+C\right), \ldots$,

$$
\begin{gathered}
\delta_{2}=z_{n}-\left(B x_{n}+C\right) \text {. Note } I=\sum_{i=1}^{n} \delta_{i}^{2} \text {, there are } \frac{\partial I}{\partial B}=0 \text { and } \frac{\partial I}{\partial C}=0 \text {. The solve is } \\
B=\frac{\sum_{i=1}^{n} x_{i} \sum_{i=1}^{n} z_{i}-n \sum_{i=1}^{n} z_{i} x_{i}}{\left(\sum_{i=1}^{n} x_{i}\right)^{2}-n \sum_{i=1}^{n} x_{i}^{2}}, C=\frac{\sum_{i=1}^{n} x_{i} \sum_{i=1}^{n} z_{i} x_{i}-\sum_{i=1}^{n} z_{i} \sum_{i=1}^{n} x_{i}^{2}}{\left(\sum_{i=1}^{n} x_{i}\right)^{2}-n \sum_{i=1}^{n} x_{i}^{2}}
\end{gathered}
$$

\section{Experiment and the result}

Experimental materials include a circular plastic sheet with $10 \mathrm{~cm}$ diameter, a few coins, needles, fine lines, mobile phone and cell phone ring, pieces of white paper, transparent tape, scissors, a pen, a scale, a stick, and sun umbrella.

(1) Experimental preparation: the pieces of white paper are cut and its size is same as that of the plastic sheet. The plastic sheet is divided into two half circle with a pen. The one of the half circle is filled as black and another is white. On the edge of the plastic sheet, there are three equal diversion points and three lines through and fixed. The other end of the three lines is tie a knot and links a stick with transparent tape.

(2) Experiment method: A few coins are put on the centre of the plastic sheet. The purpose is balance when the coins and the plastic sheet are into water. The length of the line that is between the stick and the edge of the plastic sheet is 1 meter and there are makes for 1 centimeter one mark on the line with the pen. Then a few coins are fixed under the centre of the plastic sheet.

(3) Experiment process: The experimental site is chosen in the water field in where the water is clean, clam, and deep. The stick is inserted near the river and the end cross of the stick is horizontal for the mobile phone taking photos. The line is through the cell phone ring that is fixed on the mobile phone. The purpose is that the direction of taking photos is perpendicular to the plastic sheet and for recording the distance between the plastic sheet and the interface of water and air. The mobile phone takes photos when the distance is $0,0.1 \mathrm{~cm}, 0.2 \mathrm{~cm}, 0.3 \mathrm{~cm}, \ldots$.

(4) The series photos are copied into computer and numbered as $0000,0001,0002, \ldots$ that means $0 \mathrm{~cm}, 1 \mathrm{~cm}, 2 \mathrm{~cm}, \ldots .$. The experimental scene is as figure 2.

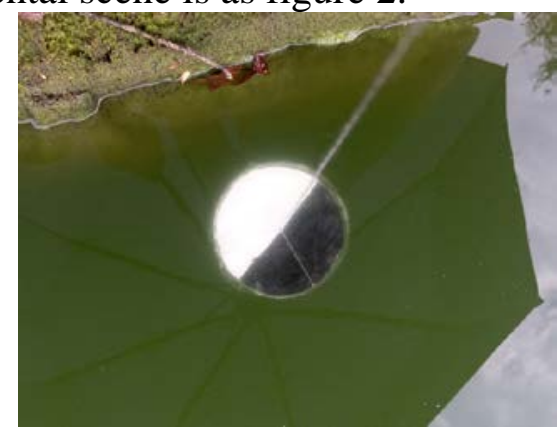

Figure 2. The scene photographed 
Images. There are 16 photos are chosen and are corresponding to the depth is $0.05 \mathrm{~cm}, 0.06 \mathrm{~cm}, \ldots$, $0.20 \mathrm{~cm}$ as shown in table 1 . Because the black half circle is not uniformity the chosen photo is from $0.05 \mathrm{~m}$ and it could be distinguished from the depth is $0.2 \mathrm{~m}$.

Table 1. The images list with different depth in water

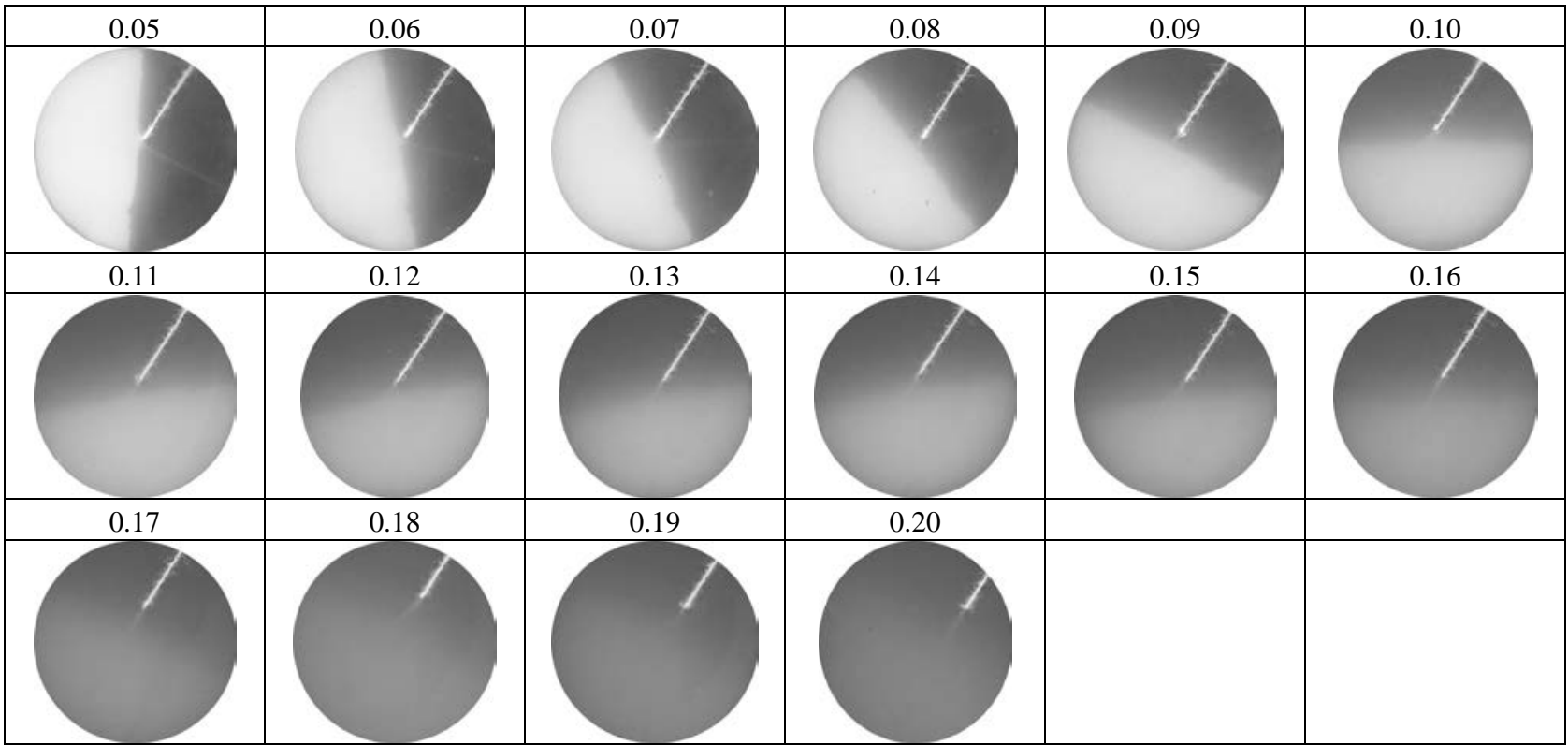

Calculation of image parameters. The image parameters of the brightness, contrast, and the product of the brightness and the contrast could be calculated by the program. The brightness, contrast, and the product of the brightness and the contrast are listed in table 2.

Table 2. The brightness, contrast, and the product of the brightness and the contrast

\begin{tabular}{|c|c|c|c|c|c|c|c|c|}
\hline$h / m$ & 0.05 & 0.06 & 0.07 & 0.08 & 0.09 & 0.10 & 0.11 & 0.12 \\
\hline bright & 191.5537 & 189.7722 & 185.3442 & 185.1480 & 183.2243 & 181.3588 & 173.4624 & 167.0811 \\
\hline contrast & 65.1383 & 60.1467 & 60.2947 & 57.8687 & 55.3756 & 50.5261 & 52.7013 & 54.3217 \\
\hline $\mathrm{b}^{*} \mathrm{c}$ & 12477 & 11414 & 11175 & 10714 & 10146 & 9163.4 & 9141.7 & 9076.1 \\
\hline$h / \mathrm{m}$ & 0.13 & 0.14 & 0.15 & 0.16 & 0.17 & 0.18 & 0.19 & 0.20 \\
\hline bright & 161.5281 & 164.1685 & 159.5215 & 156.2137 & 151.8212 & 155.7653 & 148.5606 & 147.1064 \\
\hline contrast & 55.9073 & 53.9511 & 55.9766 & 56.3286 & 56.2008 & 53.3841 & 57.0932 & 57.4849 \\
\hline b*c & 9030.6 & 8857.1 & 8929.5 & 8799.3 & 8532.5 & 8315.4 & 8481.8 & 8456.4 \\
\hline
\end{tabular}

Calculation of the fitting curve of exponential function. (1)The program for calculation of the brightness fitting curve of exponential function is programed and the brightness fitted curved of exponential function is as figure 3(a) and the fitted curve of polynomial function is as figure 3(b).

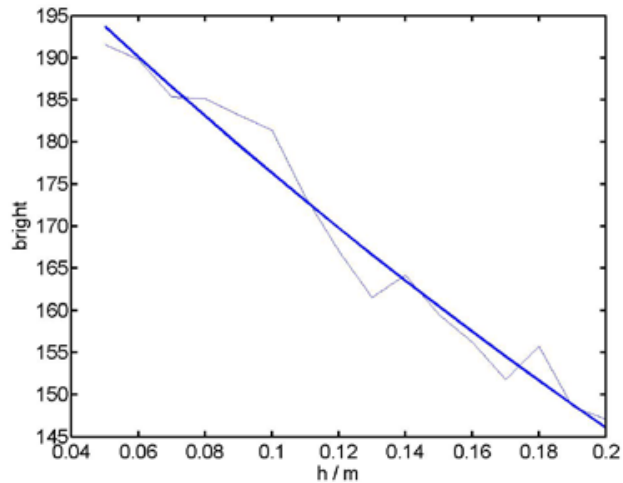

(a)

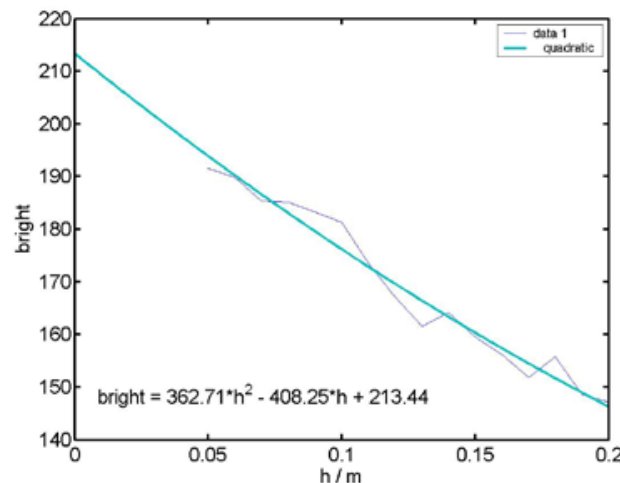

(b)

Figure 3. The brightness fitted curve. (a) exponential function; (b) polynomial function

The polynomial function of fitting curve shown in figure 3(b) is bright $=362.71 h^{2}-408.25 h+213.44$.

(2)The program for calculation of the contrast fitting curve of exponential function is programed and the slope is $B=-0.5035$, the intercept is $C=4.0940$, and the parameter is $A=59.97933$. Then the brightness fitting curve of exponential function is contrast $=59.97933 \mathrm{e}^{-0.5035 h}$. The contrast fitting curve of exponential function with the depth could be drawn and the contrast fitted curved of exponential 
function is as figure 4(a) and the fitted curve of polynomial function is as figure 4(b). The polynomial function of fitting curve shown in figure 4(b) is contrast $=1258.4 h^{2}-344.94 h+77.199$.

(3)The program is for Calculation of the product of the brightness and the contrast. The fitting curve of exponential function is drawn and the slope is $B=-2.3856$, the intercept is $C=9.4544$, and the parameter is $A=12764.2$. Then fitting curve of exponential function for the product of the brightness and the contrast is bright c contrast $=12764.2 \mathrm{e}^{-2.3856 h}$. The fitting curve of exponential function with the depth for the product of the brightness and the contrast could be drawn and the fitted curved of exponential function for the product of the brightness and the contrast is as figure 5(a) and the fitted curve of polynomial function is as figure 5(b). The polynomial function of fitting curve shown in figure 5(b) is

bright $*$ contrast $=2.4953 \times 10^{5} h^{2}-86060 h+15873$.

Analyses . (1)The variance of the bright with the fitting curve of exponential function is 6.916 and that of polynomial function is 6.864. The variance of polynomial function is less than that of exponential function.

(2)The variance of the contrast with the fitting curve of exponential function is 9.151 and that of polynomial function is 3.547. The variance of polynomial function is less than that of exponential function.

(3)The variance of the product of the brightness and the contrast with the fitting curve of exponential function is $1.4550 \times 10^{6}$ and that of polynomial function is $0.056827 \times 10^{6}$. The variance of polynomial function is much less than that of exponential function.

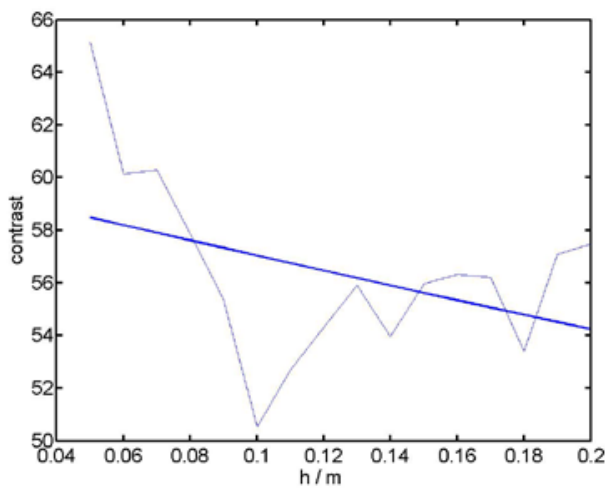

(a)

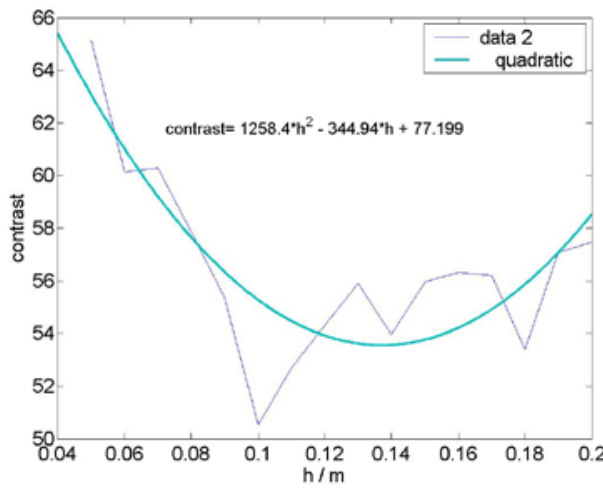

(b)

Figure 4. The contrast fitted curve. (a) exponential function; (b) polynomial function

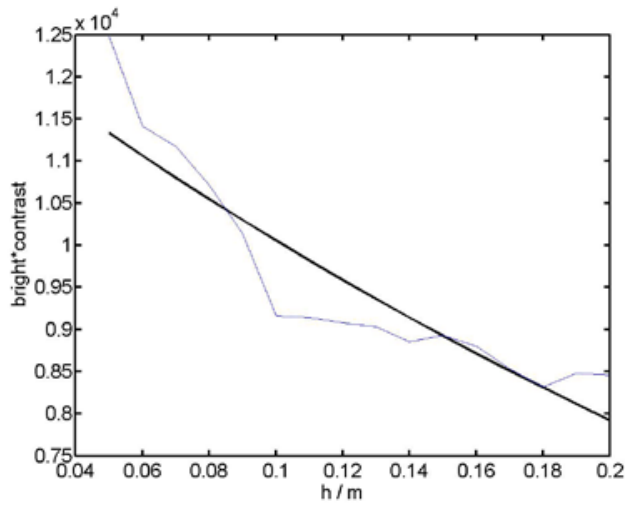

(a)

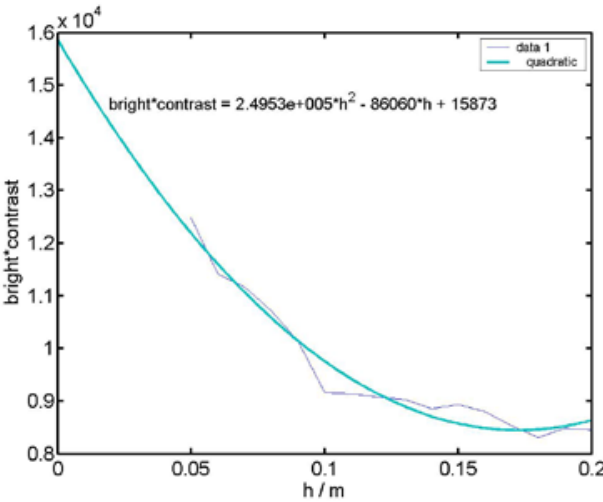

(b)

Figure 5. The fitted curve of the product of the brightness and the contrast. (a) exponential function; (b) polynomial function

\section{Conclusion}

Because the interaction between the photons and water is very complex, the transmission process is attenuation process that is like to exponential function. The reflection process is not like to exponential function. The fitted polynomial function is better than that of exponential function. The distinguished depth of object is two times of the diameter. The fitting curves of the brightness and the 
contrast with exponential function is tolerated. However, the fitting curves of the product of the brightness and the contrast with polynomial function is good.

\section{Acknowledgements}

This work was financially supported by the State Key Laboratory of Satellite Ocean Environment Dynamics (Second Institute of Oceanography, SOA) (SOED1503).

\section{References}

[1] Ling Zaiying. Modeling the bidirectional reflectance distribution function of water based on Monte Carlo simulation [D]. Master's degree dissertation of Zhejiang University. 2007

[2] Hu zhigang. The research of underwater 3D positioning technique based on binocular stereo vision [D]. Master's degree dissertation of Zhongguo ocean University. 2011

[3] Huang Ping. The research of the theory and experiment for Laser underwater object detection with Monte Carlo algorithm [D]. Master's degree dissertation of Huazhong University of science and technology. 2011

[4] Li Huiyuan. The Monte Carlo simulation of Laser underwater object detection [D]. Master's degree dissertation of Southwest Jiaotong University. 2011

[5] Li Shengfu, Chen Guanghua, Wang Rongbo, et al. Monte Carlo based angular distribution estimation method of multiply scattered photons for underwater imaging [J]. Optics Communications. 2016, 381: 43-47

[6] Li Xinlu. Analysis Simulation of the three defects and Research of the Image Enhancement Method for Underwater Imaging [D]. Master's degree dissertation of Changzhou University. 2017 